\title{
Trabectedin expands treatment options for some forms of advanced soft tissue
}

\section{sarcoma}

I n the fall of 2015, the United States joined the growing list of countries in which trabectedin, a novel form of chemotherapy, is approved for the treatment of certain types of advanced soft tissue sarcoma. The drug, a synthetic derivative of a compound originally isolated from a sea squirt, has a complex mechanism of action that distinguishes it from other cytotoxic drugs, allowing it to target both the tumor and its microenvironment.

Based on the results of a randomized, open-label, multicenter phase 3 clinical trial, trabectedin received regulatory approval for the treatment of unresectable or metastatic liposarcoma or leiomyosarcoma. These represent 2 of the most common types of sarcoma occurring in the fat cells and smooth muscle cells, respectively, and patients have few effective therapeutic options. Trabectedin is approved for use in patients who previously received anthracyclinebased chemotherapy.

The ET743-SAR-3007 trial enrolled patients at 85 sites in 4 different countries from May 27, 2011 to September 16, 2013. Patients were aged 15 years or older, with unresectable, locally advanced or metastatic liposarcoma or leiomyosarcoma, had been previously treated with either a combination of an anthracycline and ifosfamide or an anthracycline plus 1 or more additional cytotoxic chemotherapy regimens. Eligible patients were also required to have adequate bone marrow, renal and liver functions, and an Eastern Cooperative Oncology Group performance status of 1 or lower. Those with known central nervous system metastases, myocardial infarction within the 6 months before enrollment, or New York Heart Association class II or greater heart failure were excluded from the study.

In all, 518 patients were randomly assigned in a 2:1 ratio to receive either trabectedin at a starting dose of $1.5 \mathrm{mg} /$ $\mathrm{m}^{2}$ or dacarbazine at a starting dose of $1 \mathrm{~g} / \mathrm{m}^{2}$. The study drug was administered on day 1 of each 21-day treatment cycle until disease progression or unacceptable toxicity. Trabectedin was administered over 24 hours as an intravenous infusion, following premedication with intravenous dexamethasone $20 \mathrm{mg}$, and dacarbazine was administered as a $20-120$-minute intravenous infusion.

Tumor response was assessed by radiographic imaging of the chest, abdomen, and pelvis every 6 weeks for the

\section{What's new, what's important}

The US Food and Drug Administration approved trabectedin for the treatment of unresectable or metastatic liposarcoma and leiomyosarcoma that have been treated with anthracycline. Trabectedin has a unique mechanism of action whereby it causes DNA damage and affects transcription regulation in a promoterand gene-specific manner. In addition, it alters the tumor microenvironment. The drug was approved on the basis of findings in a randomized controlled study showing that it improved progression-free survival (4.2 months vs 1.5 months for dacarbazine). The FDA-recommended dose is $1.5 \mathrm{mg} / \mathrm{m}^{2}$ as a 24 -hour intravenous infusion, every 3 weeks. Patients were premedicated with dexamethasone $20 \mathrm{mg} \mathrm{IV}, 30$ minutes before each infusion.

Trabectedin carries a boxed warning to alert health care practitioners to the risk of severe and fatal blood infections, rhabdomyolysis, hepatotoxicity, extravasation, tissue necrosis, and heart failure and to caution medical practitioners to advise women of the potential risk to a developing fetus and not to breastfeed while taking the drug. The most common side effects among participants in the study who received trabectedin were nausea, fatigue, vomiting, diarrhea, constipation, decreased appetite, shortness of breath, headache, peripheral edema, neutropenia, thrombocytopenia, anemia, elevated liver function tests.

Although the survival benefit with trabectedin is modest, it offers a new treatment option for patients with liposarcoma and leiomyosarcoma.

- Jame Abraham, MD, FACP (abrahaj5@ccf.org)

first 36 weeks and then every 9 weeks thereafter until disease progression, subsequent anticancer therapy, or patient death occurred. The primary endpoint was overall survival (OS), with secondary endpoints of progression-free survival (PFS), time to progression, objective response rate (ORR), and duration of response.

Baseline demographics and disease characteristics were well balanced. The patients had been heavily pretreated more than $80 \%$ had received at least 2 prior lines of systemic therapy, more than $90 \%$ had previous surgery, and half had prior radiation therapy.

After 329 PFS events, median PFS was significantly

Report prepared by Jane de Lartigue, PhD. JCSO 2016;14:189-191. (02016 Frontline Medical Communications. doi: 10.12788/ jeso.0253. 


\section{Mechanism of action: trabectedin}

\section{A dual blow to tumor and microenvironment}

Trabectedin is a synthetic compound originally derived from a marine tunicate that, though classed as a cytotoxic chemotherapy, has a novel mechanism of action that distinguishes it from other alkylating agents. Due to the double helix shape of the DNA, with strong hydrogen bonds that pull the 2 backbone strands together, so-called major and minor grooves form along the length of these strands. These grooves have important implications for DNA function because they impact the ability of proteins involved in transcription and repair to bind to the DNA.

Trabectedin is thought to bind to the minor groove of the DNA, which causes the DNA helix to bend toward the major groove and induces DNA damage that triggers the nucleotide excision repair (NER) pathway. Because bound trabectedin also protrudes from the DNA helix, it blocks several DNA-binding molecules, including transcription factors and proteins involved in the NER pathway. In addition to interfering with transcription regulatory pathways, this prevents the trabectedin-induced DNA damage from being repaired and results in the formation of double-strand breaks, which subsequently lead to cell death.

More recently, several studies have suggested that trabectedin's mechanism of action extends beyond these effects on tumor cells and that it also impacts normal host cells of the tumor microenvironment. These studies have suggested that it affects blood monocytes and tumor-associated macrophages in particular, as a result of its ability to activate the caspase- 8 pathway downstream of the tumor necrosis factor-related apoptosisinducing ligand membrane receptor. It has also been reported to affect the production of protumoral inflammatory cytokines. improved in the trabectedin group (4.2 vs 1.5 months in the dacarbazine arm) and trabectedin treatment resulted in a $45 \%$ reduction in the risk of disease progression or death. PFS benefit was observed across all preplanned subgroups and in both sarcoma subtypes and was confirmed by an independent review of radiographic PFS. Subsequent anticancer therapy was used less in the trabectedin group and median time to initiation of this therapy was also significantly longer.

ORR was also significantly higher in trabectedin-treated patients (9.9\% vs 6.9\%) and, although there were no complete responses in either group, trabectedin resulted in more partial responses and stable disease as best response. Median duration of response was 6.5 months in the trabectedin arm, compared with 4.2 months in the dacarbazine arm, and median duration of stable disease was also longer with trabectedin treatment. The clinical benefit rate was $34 \%$ compared with $19 \%$ in the 2 arms.

Although no significant improvement in OS was observed, the OS results favored the trabectedin arm, with an overall 13\% reduction in the risk of death. OS results may have been confounded by post-trial chemotherapy and median OS in the dacarbazine group, which was reported to exceed historical expectations.

The adverse event (AE) profile of both drugs was consistent with previously reported safety and toxicity profiles, most commonly grade 1-2 in severity. The most common AEs experienced in the trabectedin arm included nausea, fatigue, vomiting, diarrhea, constipation, decreased appetite, dyspnea, headache, peripheral edema, neutropenia, thrombocytopenia, anemia, elevated liver enzymes, and decreased albumin levels. The proportion of patients 
who died within 60 days of the first dose was similar in the 2 treatment arms $(7.1 \%$ and $5.8 \%$ for trabectedin and dacarbazine, respectively), though treatment-related deaths occurred only in the trabectedin arm.

The recommended dose of trabectedin is $1.5 \mathrm{mg} / \mathrm{m}^{2}$ administered over 24 hours through a central venous line every 21 days in patients with normal bilirubin and aspartate aminotransferase (AST) or alanine aminotransferase (ALT) levels $\leq 2.5$ times the upper limit of normal (ULN).

Trabectedin carries a boxed warning, alerting health care practitioners to the risk of severe and fatal blood infections, rhabdomyolysis, hepatotoxicity, extravasation, tissue necrosis, and heart failure and cautions medical practitioners to advise women of the potential risk to a developing fetus and not to breastfeed while taking the drug.

Trabectedin is marketed as Yondelis by Janssen Pharmaceuticals Inc. The prescribing information advises

\section{References}

1. US Food and Drug Administration. FDA approves new therapy for certain types of advanced soft tissue sarcoma. US Food and Drug Administration Web site. http://www.fda.gov/NewsEvents/ Newsroom/PressAnnouncements/ucm 468832.htm. Released October 23, 2015. Accessed March 12, 2016.

2. Demetri GD, von Mehren M, Jones RL, et al. Efficacy and safety of trabectedin or dacarbazine for metastatic liposarcoma or leio- that neutrophil count, creatinine phosphokinase levels, and liver function tests (defined as ALT, AST, total bilirubin, and alkaline phosphatase levels) should be assessed before each dose of trabectedin, and left ventricular ejection fraction (LVEF) should be assessed before starting on this drug and at 2-3 month intervals thereafter. Trabectedin treatment should be withheld for neutrophil counts $<1,500$ cells $/ \mu \mathrm{L}$ on the day of dosing, serum CPK levels $>2.5$ times ULN, and LVEF below the lower limit of normal.

Elevated liver function tests should be managed with treatment interruption, dose reduction, or permanent discontinuation reflecting the severity and duration. Trabectedin should be discontinued in the event of lifethreatening or prolonged, severe neutropenia in the previous cycle, for rhabdomyolysis, and for symptomatic cardiomyopathy or persistent left ventricular dysfunction that doesn't recover to the lower limit of normal within 3 weeks.

myosarcoma after failure of conventional chemotherapy: results of a phase III randomized, multicenter clinical trial. J Clin Oncol. 2016;34(8):786-793.

3. Yondelis (trabectedin) injection, for intravenous use. Prescribing Information. Janssen Pharmaceuticals, Inc. https://www.janssenmd. com/pdf/yondelis/YONDELIS_PI.pdf. Issued October 2015. Accessed March 12, 2016. 